\title{
Creación musical colectiva: análisis de prácticas pedagógicas disruptivas en Educación Superior
}

\author{
Almudena Ocaña-Fernández¹; Ramón Montes-Rodríguez²; María-Luisa Reyes-López
}

Recibido: 2 de enero de 2020 / Aceptado: 8 de junio de 2020

Resumen. Este texto se centra en un proceso de investigación llevado a cabo en un proyecto de creatividad musical colectiva en el plan de formación inicial de 142 futuros docentes de Educación Primaria. El objetivo del estudio es comprender cómo se conforman sus procesos de aprendizaje durante su paso por una experiencia musical creativa y potencialmente disruptiva. Para ello hemos utilizado una metodología de estudio de caso, que mediante el análisis de contenido de los informes del alumnado, las grabaciones de clases y diarios de investigación nos ha permitido comprender las perspectivas del estudiantado desde una mirada profunda y reflexiva.. Los principales resultados señalan que utilizar elementos creativos artísticos y musicales en Educación Superior resulta disruptivo para los estudiantes, que el alumnado mayoritariamente pasa de estados iniciales de incertidumbre y confusión a estados de motivación y de compromiso hacia la propia práctica, y que este tipo de proyectos que fomentan la creatividad en la Universidad a través de otros lenguajes como la música dinamizan y potencian aprendizajes más reales y significativos en docentes en formación.

Palabras clave: Creatividad; Formación del profesorado; Educación Musical; Innovación Educativa; Investigación cualitativa.

\section{[en] Collective musical creation: analysis of disruptive pedagogical practices in Higher Education}

\begin{abstract}
This text focuses on a research project carried out in collaboration with a teaching project that uses collective musical creativity in the initial training of 142 future teachers of Primary Education. The objective of the study is to understand how the students' learning processes are shaped during their passage through a creative and potentially disruptive musical experience that took place during one semester. For this we used a case study methodology which, through content analysis of student's own reports, video recordings of classes and research diaries, allowed us to understand the views of these students from a deeper and more reflective perspective. The main results indicate that using artistic and musical creative elements in Higher Education is innovative and disruptive for students. We found that in this experience most students go from initial states of uncertainty and confusion to states of motivation and engagement. Also, we can see how this type of project fosters creativity in the University through other languages such as music to stimulate and enhance real and meaningful learning in future teachers.
\end{abstract}

Keywords: Creativity; Teacher Training; Music Education; Educational Innovation; Qualitative Research

Sumario. 1. Introducción. 2. Entre la innovación, la disrupción y la creatividad 3. Preguntas de investigación y propósito del estudio. 4. Metodología. 5. Resultados. 6. Discusión y conclusiones. 7. Limitaciones y futuras líneas de investigación. 8. Referencias bibliográficas

Cómo citar: Ocaña-Fernández, A., Montes-Rodríguez, R. y Reyes-López, M.L. (2020) Creación musical colectiva: análisis de prácticas pedagógicas disruptivas en Educación Superior. Revista Electrónica Complutense de Investigación en Educación Musical, 17, 3-12.

Facultad de Ciencias de la Educación. Universidad de Granada (España)

E-mail: aocafer@ugr.es

Facultad de Ciencias de la Educación. Universidad de Granada (España)

E-mail: ramontes@ugr.es

Facultad de Ciencias de la Educación. Universidad de Granada (España)

E-mail: mlreyes@ugr.es 


\section{Introducción}

La escuela del siglo XXI se encuentra inmersa en una sociedad de vertiginosos cambios e incesantes procesos de innovación. Una sociedad caracterizada por fenómenos como la globalización, el neoliberalismo o el auge de las TIC donde la naturaleza del conocimiento es más bien efímera. Una sociedad líquida (Bauman, 2007) y knowmádica (Moravec, 2013) caracterizada por la fragmentación, la incertidumbre, lo inestable, y por las aceleradas transformaciones sociales y culturales consecuencia del desarrollo tecnológico.

Es en este contexto donde se hace necesario plantear el aula como espacio pedagógico flexible y diverso, donde el protagonista sea el estudiante (Pérez Gómez, 2012). Una escuela comprensiva, democrática, plural, ideológica y culturalmente independiente, abierta y orientada a conseguir el desarrollo integral de la personalidad. Y donde los estudiantes investiguen, reflexionen, apliquen y compartan conocimiento, poniéndose en juego formas de aprendizaje interactivas, personalizadas, colaborativas, creativas e innovadoras que los mantengan implicados (Dede, 2007); y que posibilite a su vez, que docentes y estudiantes sean coaprendices y coeducadores, olvidando y dejando atrás la dualidad enseñanza-aprendizaje y docente-aprendiz más propia de la escuela de siglos pasados (Moravec, 2011).

A su vez, se hace necesario un cambio de mirada, una nueva concepción del docente como un profesional que ya no solo se define por su capacidad de transmitir conocimientos y evaluar (Pérez Gómez, 2008) sino al que se le exigen unas competencias más complejas para la tarea de provocar, acompañar, orientar y cuestionar el aprendizaje de los estudiantes y para prepararlos para la vida (Perrenoud, 2012). Donde el pensamiento creativo, emergente, flexible y estratégico será un elemento angular para tener en cuenta en la escuela y, por ende, en la formación de los futuros docentes (Murillo-Ribes, Riaño-Galán, y Berbel-Gómez, 2018).

Este cambio de mirada nos obliga a considerar el aula universitaria como un espacio en el que se ponen en juego ecologías de saberes (Santos, 2010) no solo disciplinares sino también prácticos y experienciales (Contreras, 2013), donde la movilización y construcción de dichos saberes necesita de procesos de aprendizaje reflexivos, cooperativos y creativos que generen el intercambio de ideas, de opiniones y de puntos de vista donde la jerarquía de roles entre docentes y estudiantes desaparezca. En esta línea este artículo presenta un proceso de investigación ligado a una práctica docente en el contexto de la Educación Superior desde presupuestos cercanos a lo que se entiende por disrupción educativa, con el objetivo de generar formas de aprendizaje alternativas en el campo de la Educación Musical.

\section{Entre la innovación, la disrupción y la creatividad}

$\mathrm{Al}$ indagar en los orígenes del concepto disrupción ligado a lo educativo observamos que este aparece vinculado a aspectos puramente tecnológicos (Bower y Christensen, 1995). No obstante, en los últimos años varios autores han presentado nuevos planteamientos más creativos que toman algunos elementos de la disrupción pensada desde lo tecnológico en espacios de Educación Superior (Anderson y McGreal, 2012; Hutchings \& Quinney, 2015; Al-Zahrani, 2015), pero que transcienden esta concepción para poner el foco en la importancia de los procesos de creación compartida, entendiendo la creatividad como la posible nueva disrupción del siglo XXI. Al revisar la literatura observamos un desplazamiento a posiciones más ligadas a cambios culturales que consideran la disrupción como un desafío de lo establecido, como procesos contrahegemónicos y de un cierto hartazgo para con lo tecnológico (San Pedro, 2018; Quilty, 2017). De un modo similar, Hesse-Biber y Leavy (2006) redefinieron la disrupción educativa como el hecho de irrumpir y romper con las formas tradicionales de saber y conocer para crear nuevos y valiosos significados; y otros como Adams, Bell y Griffin (2007) la conceptualizan como jugar un rol principal en el desarrollo de un tipo de pensamiento crítico y una perspectiva ética inclusiva que apoye el respeto por la diferencia. Entre las características que debe reunir un proceso pedagógico potencialmente disruptivo, Hedberg y Freebody (2007) señalan que: 1) produce cambios observables en la enseñanza/aprendizaje; 2) desarrolla actividades que requieren secuencias pedagógicas genéricas que pueden ser aplicadas en distintas áreas de lo curricular, favoreciendo así el aprendizaje interdisciplinar; 3) anima a utilizar distintos modos de representación más allá de lo textual; 4) activa el compromiso y la motivación; 5) favorece interacciones que promueven la construcción social y significativa del conocimiento; 6) la evaluación se entiende como un proceso; y 7) puede utilizar repositorios de tipo digital para potenciar la expansión del conocimiento.

Para esta investigación, la disrupción es entendida desde posiciones más cercanas a lo creativo que a lo tecnológico, pues en una Educación Superior acostumbrada a lo vertical y lo directivo, es el proceso de construcción colaborativa del conocimiento mediante prácticas creativas el que funciona como algo disruptivo. Y esto, como podemos observar en la literatura académica, es algo que no sucede frecuentemente. Bautista, Yau y Wong (2016) manifiestan que los centros escolares que optan por metodologías disruptivas necesitan maestros formados en lo tecnológico, lo creativo o el trabajo en equipo, pero que encuentran poca motivación en realizar prácticas sonoras/musicales por la escasez de experiencias musicales creativas previas.

La innovación pedagógica en Educación Musical ha estado protagonizada por las TIC en estas últimas décadas, con investigaciones centradas en probar la eficacia educativa de lo digital en lo musical (Lorenzo-Quiles, Vílchez-Fernández y Herrera-Torres, 2015), y al igual que en educación en general, las iniciativas TIC han acaparado el centro del discurso cuando se hablaba de disrupción. En el contexto de la Educación Superior se han realizado trabajos sobre Educación Musical y metodologías flipped classroom, donde la innovación se situaría en la creación 
de materiales audiovisuales alojados en línea (Parra y Gutiérrez, 2017; García-Gil y Cremades-Andreu, 2019); sobre gamificación a través de las tecnologías y propuestas de innovación a través de metodologías basadas en el juego (Carrión, 2019); sobre herramientas específicas como Soundcool que permiten procesos de creación sonora en tiempo real y que facilitan los procesos de interacción, escucha atenta y aprendizaje colaborativo a través de la experimentación con el sonido (Murillo-Ribes, Riaño-Galán y Berbel-Gómez, 2018); o sobre el desarrollo de competencias digitales e interdisciplinares a través de experiencias disruptivas centradas en el concepto de paisaje sonoro (Botella, Hurtado y Ramos, 2018).

Pero también encontramos trabajos académicos que hablan de innovación y de propuestas disruptivas en Educación Musical desde posicionamientos alejados de lo tecnológico y más cercanos al de nuestro trabajo. En ese sentido, Vass (2019) realiza una investigación sobre la metodología Kokas para innovar en Educación Musical a través del movimiento, de la importancia del cuerpo y del valor de las reflexiones y del diálogo de los participantes (corporal y verbal), siendo una de sus aportaciones más significativas la de plantear la necesidad de construir un aprendizaje que vaya más allá del conocimiento y praxis que puede ofrecer el lenguaje verbal o escrito. Kenny (2018), también desde posicionamientos cualitativos, planteó una investigación sobre iniciativas corales en la comunidad universitaria, donde se señala el potencial del lenguaje musical para desarrollar la capacidad de liderazgo y empoderamiento en comunidades desfavorecidas. Y Minors et al. (2017), centrándose en el concepto de nuevas creatividades, explora distintas experiencias de Educación Musical en Educación Superior para evidenciar que es necesario contar con la voz de los estudiantes para poder así mejorar las prácticas educativas y los planes curriculares.

A partir de estas experiencias y del conocimiento que se vierte de ellas, aquí se presenta una investigación que recoge el análisis de una propuesta de creación musical colectiva en Educación Superior.

\section{Preguntas de investigación y propósito del estudio}

Esta investigación nace por la necesidad de reflexionar de manera sistemática sobre el desarrollo de nuestra práctica docente universitaria en torno a dos elementos clave: la creatividad y la investigación dentro del aula.

El objetivo principal del estudio es comprender desde una mirada profunda y reflexiva cómo se conforman los procesos de aprendizaje de 142 futuros docentes durante su paso por esta experiencia musical creativa y potencialmente disruptiva. Para ello, formulamos una serie de preguntas de investigación etic (Stake, 1998), que, dada la importancia de lo emergente en la investigación cualitativa, han sido condicionadas de forma emic durante toda la fase de análisis de los datos, siendo estas las preguntas finales que han guiado el proceso:

1. ¿Qué elementos caracterizan a esta práctica pedagógica disruptiva?

2. ¿Qué aprende el alumnado y el profesorado al desarrollarla?

3. ¿Qué papel juega la creatividad musical durante todo el proceso?

\section{Metodología}

Para el diseño de investigación se ha partido de un paradigma metodológico de corte cualitativo, en concreto, del estudio de caso (Yin, 1994; Stake, 1998; Simons, 2011) que nos permite reflexionar, comprender y analizar en profundidad procesos complejos de innovación educativa desde una mirada interpretativa.

\subsection{Participantes}

El proyecto fue desarrollado en la asignatura de "Educación Musical" del Grado de Educación Primaria de la Universidad de Granada. Esta materia es obligatoria y se imparte en el segundo semestre del tercer curso. La experiencia se llevó a cabo con dos grupos de aproximadamente 70 personas, para un total de 142 estudiantes. Para muchos, supuso el primer contacto con la Educación Musical, y para la gran mayoría, los seis créditos de esta asignatura son la única oportunidad que tienen de aproximarse a la música. Sus características son bastante similares en ambos grupos y, por lo tanto, los datos recogidos se han tratado sin tener en cuenta estos agrupamientos como elemento de heterogeneidad. Existe cierto equilibrio entre el número de varones y mujeres, siendo el porcentaje de estas últimas ligeramente superior (55\%). Y podemos decir que se trata de estudiantes con escasa o nula formación musical, ya que menos del $15 \%$ de los participantes señalan tener conocimientos musicales más allá de las clases recibidas durante su paso por la enseñanza obligatoria.

En general, las expectativas que muestran al inicio sobre la asignatura están relacionadas con la adquisición de una formación teórica y práctica sobre las cuestiones ligadas a la experiencia musical (leer música, tocar, cantar), encontrándose el valor educativo de la música y sus posibilidades en segundo plano. Cuando se les pregunta acerca de las características que debe tener un docente y sobre el valor de la creatividad, nos hablan de empatía, de afecto, paciencia, implicación, interés y motivación, y vinculan la creatividad musical a la figura del músico experto. Pero no observamos que conozcan el potencial educativo de utilizar prácticas de creatividad musical en el aula. 


\subsection{Descripción de la práctica educativa}

La propuesta creativa consiste en la realización de una composición musical a partir de un itinerario de creación colectiva guiado utilizando como herramientas básicas el movimiento y el sonido. Los objetivos que se pretenden con el desarrollo de esta actividad son tres principalmente y están relacionados con el desarrollo de competencias docentes del profesorado en formación que consideramos esenciales: a) promover el aprendizaje autónomo y reflexivo; b) generar un espacio de trabajo colaborativo real; e c) identificar estrategias metodológicas que nos permitan aprender y enseñar creativamente. Paralelamente se atienden objetivos vinculados con la formación musical que -como hemos mencionado- es escasa o nula en este grupo de estudiantes: a) reflexionar sobre los procesos creativos musicales y sus implicaciones educativas; b) utilizar la música como medio de comunicación alternativo; y c) comprender, a partir de la experiencia, los parámetros del sonido y los elementos de la música.

Esta propuesta se inicia tras haber trabajado previamente la escucha e interpretación creativa con los estudiantes a través de actividades que implican la audición activa, el movimiento, la voz y la expresión instrumental.

La creación musical está acompañada en todo momento de un proceso de reflexión compartida a través de la realización de un portafolio grupal para recoger el proceso de creación y reflexionar sobre el aprendizaje, sus certezas e incertidumbres. Al finalizar y utilizando la información recogida en ese portafolio elaboran un informe de investigación donde describen y reflexionan para conectar los y reflexionan para conectar los conocimientos teóricos y prácticos de la asignatura. Este trabajo supone un momento en el que entran en diálogo los saberes disciplinares, prácticos y experienciales. A continuación, describimos la secuencia metodológica que se desarrolla durante cuatro sesiones de una hora.

\section{SESIÓN 1: CREACIÓN DEL AUTÓMATA}

a. Ideas previas del grupo sobre el concepto de autómata (Lluvia de ideas).

b. Descripción del autómata como una máquina que imita la figura y los movimientos de un ser animado utilizando un mecanismo de engranajes cerrados.

c. Visionado de ejemplos de autómatas en Youtube (https://www.youtube.com/watch?v=uz8TV7gkeT0).

d. Por grupos de 4-6 personas y tomando como idea inspiradora el concepto de autómata crean una secuencia de movimientos colectivos conectados entre sí. Para la creación de este "autómata" deben explorar diferentes elementos relacionados con el cuerpo y el espacio como formaciones, niveles, direcciones, trayectorias, etc.

e. Presentación a la clase de cada una de las propuestas y análisis de sus elementos y de las dificultades en el proceso creativo. Este momento sirve para evaluar las propuestas, pudiendo sugerirse alguna modificación desde el grupo-clase.

\section{SESIÓN 2: SONORIZACIÓN DEL AUTÓMATA I}

a. Utilizando percusión corporal y/o sonidos vocales y teniendo en cuenta los parámetros sonoros (altura, timbre, intensidad y duración) crean una secuencia musical que acompañe a los movimientos del autómata.

b. Presentación a la clase y evaluación de la propuesta.

\section{SESIÓN 3: SONORIZACIÓN DEL AUTÓMATA II}

a. Se realiza una traducción de las sonoridades realizadas por el cuerpo y la voz al instrumental Orff. Cada grupo debe buscar un instrumento que por sus características permita reproducir el sonido que realizaban en la propuesta anterior.

b. Se interpreta la secuencia musical con estos instrumentos prescindiendo del movimiento. Este es el momento en el que se realiza una traducción de la experiencia corporal en movimiento a otra más estática, limitada al uso de los instrumentos.

c. Presentación a la clase y evaluación de la propuesta.

\section{SESIÓN 4: COMPOSICIÓN OBRA MUSICAL}

a. A partir de la idea musical obtenida a través del proceso anterior se trabaja la composición de una obra musical utilizando las técnicas de repetición, variación y contraste.

b. Una vez finalizada la composición cada grupo escribe su obra utilizando códigos de grafía no convencional.

c. Presentación a la clase y evaluación de la propuesta.

\subsection{Técnicas de recogida de datos}

Para la recogida de información se han utilizado distintas técnicas de observación participante como los diarios compartidos del investigador/docente o los registros sistemáticos en audio, previamente negociados, de los discursos de los participantes durante las clases (Tabla 1). Para el registro de observaciones se han tenido en cuenta las macrocategorías de análisis. También se han analizado los informes del alumnado (construidos a partir de la información recogida en su portafolio tal y como se ha explicado con anterioridad) y los elementos creativos y artísticos presentes en sus producciones musicales finales. 
Tabla 1. Técnicas de Recogida de Información

\begin{tabular}{|l|l|}
\hline \multicolumn{1}{|c|}{ Técnica de recogida } & \multicolumn{1}{c|}{ Cuantía } \\
\hline Diarios del investigador (PD) & 2 diarios y 1 registro compartido \\
Grabación de las clases & 8 sesiones de entre 90 y 120 minutos \\
$\begin{array}{l}\text { Informes de investigación del alumnado } \\
\text { (ESTxG1-2) }\end{array}$ & 26 informes grupales. 20 páginas de media \\
\hline
\end{tabular}

En cuanto a temas éticos, queremos destacar que los estudiantes fueron informados desde el primer momento del desarrollo de la investigación, justificando la necesidad que teníamos como docentes-investigadoras de evaluar de forma comprensiva los procesos pedagógicos disruptivos que se iban a implementar durante la asignatura. Tras informar de que el anonimato y la confidencialidad estaban garantizados se obtuvo el consentimiento informado de los participantes. También se negoció y realizó la triangulación de la información obtenida a través de distintas sesiones en las que se les presentaron los resultados de la investigación.

\subsection{Técnicas de análisis de datos}

El análisis cualitativo de los datos se ha caracterizado por ser interactivo e iterativo, operando en una espiral de profundización (Strauss y Corbin, 2002) durante toda la investigación. Estos datos fueron estudiados mediante el análisis de las temáticas del discurso y la comparación y triangulación constante de la información obtenida de las diferentes fuentes analizadas (Maxwell, 2012). Comenzamos realizando un sistema de categorías a partir de la revisión de la literatura científica y posteriormente este sistema, exclusivamente deductivo, fue cambiando durante el proceso de investigación con los datos emergentes de los discursos de los participantes. Surgieron distintas categorías inductivas que han ido modificando y completando el sistema inicial, con la precaución de no duplicarlas, pero con la apertura de ser un sistema de categorías no exclusivo, es decir, que una misma narrativa puede formar parte de distintas categorías. Para ello, hemos tenido en cuenta las orientaciones de Simons (2011) que alerta del peligro de que la categorización se torne mecánica y formulista, estancándose en un nivel descriptivo y en la que los códigos se consideren como entes cerrados. Así, a lo largo de la categorización se ha mantenido una actitud de apertura a lo emergente y al cambio a medida que se han ido examinando las distintas fuentes de datos y se ha crecido en la comprensión de un proceso con muchas aristas.

Tabla 2. Sistema detallado de categorías (nodos) elaborados a partir del análisis

\begin{tabular}{|l|l|}
\hline \multicolumn{1}{|c|}{ Lo estructural } & \multicolumn{1}{c|}{ Lo relacional } \\
\hline $\begin{array}{l}\text { Roles profesor/alumno } \\
\text { Cambios espaciales } \\
\text { Cambios temporales }\end{array}$ & $\begin{array}{l}\text { Relación dialógica con el profesorado } \\
\text { Trabajo en grupo o Trabajo colectivo / La bús- } \\
\text { queda de los consensos } \\
\text { Emociones/Sentimientos compartidos } \\
\text { Construcción compartida, colectiva y crítica } \\
\text { del conocimiento }\end{array}$ \\
\hline \multicolumn{1}{|c|}{ Lo personal } & \multicolumn{1}{|c|}{ Lo musical / Lo creativo } \\
\hline $\begin{array}{l}\text { Autonomía/Responsabilidad/Toma de de- } \\
\text { cisiones } \\
\begin{array}{l}\text { Motivación y capacidad de liderazgo } \\
\text { Empatía con la otredad }\end{array}\end{array}$ & $\begin{array}{l}\text { La música como lenguaje/como mediación } \\
\text { La producción musical/ La composición como } \\
\text { elemento disruptivo } \\
\text { Incertidumbre en los procesos creativos }\end{array}$ \\
\hline
\end{tabular}

Por otro lado, el análisis siempre ha ido acompañado de la triangulación entre las distintas fuentes (observaciones, diarios e informes de investigación de los estudiantes), así como de debate interno entre equipo de investigación y participantes por medio de la de negociación de los informes. De modo parecido, en la categorización, análisis y discusión de los datos por parte del equipo investigador, han intervenido las dos docentes de la asignatura y un investigador externo que ha realizado un proceso de contraste y validez del sistema de categorías, mediante su comparación, puesta en común, debate y acuerdo. Para llevar a cabo este trabajo se ha utilizado como soporte tecnológico el software NVivo.

\section{Resultados}

A continuación, presentamos los hallazgos más relevantes que dan respuesta, en relación con el sistema de categorías generado, a las preguntas planteadas. No realizamos un análisis cuantitativo de la presencia de cada una de las 
categorías porque el valor numérico de estas no aporta ninguna información de calidad para dar respuesta a nuestras preguntas de investigación.

Entre los elementos que caracterizan la propuesta como disruptiva podemos señalar, en primer lugar, que se observan cambios significativos en los procesos de enseñanza-aprendizaje entre los que destaca, desde una visión de "lo estructural", la disolución de los roles tradicionales de estudiantes y docentes. Observamos que los estudiantes viven su paso por esta práctica de creación musical colectiva como algo transformador, que rompe con lo anterior y aumenta la implicación en su proceso formativo generando así entornos de aprendizaje más democráticos. Esta dinámica supone un cambio de posición, en el que se desdibujan los roles clásicos de docente y aprendiz, donde ambas partes muestran sus incertidumbres, poniendo sobre la mesa sus miedos y dilemas, y en el que los contextos adquieren una mayor importancia, tanto en lo espacial como en lo temporal, ya que en ellos se generan zonas de confianza bidireccional que minimizan las relaciones de poder y promueven la horizontalidad.

Llevamos toda una vida estudiando con un fin, presentarnos a un examen y aprobarlo, cuando nos quitan de la rutina el cambio es chocante y a la vez asusta cuando piensas que no tienes ni idea del tema. (EST3G1)

Cada día en el aula supone un nuevo reto, no saber qué va a suceder es lo que me hace no perder la ilusión por enseñar. Aunque en mi cabeza hay una estructura, estoy dispuesta a romperla en función de lo que surja por parte de los estudiantes. (PD2)

Observamos también cómo el espacio se reconfigura y se transforma para facilitar el trabajo en grupo. El uso del aula deja de ser convencional, al igual que la gestión de los tiempos, un obstáculo habitual en procesos de naturaleza creativa, que también observamos que se estructura con mayor flexibilidad. En la mayoría de las ocasiones se necesita más tiempo del que está delimitado en el horario de la asignatura, lo que obliga a los estudiantes a entender que su proceso formativo no está condicionado exclusivamente por aquello que sucede dentro de las paredes que delimitan la clase, por lo que finalmente el aprendizaje se expande más allá del espacio-aula.

En relación con "lo personal", observamos una mayor implicación del estudiante en su propio proceso de aprendizaje. Se convierten en protagonistas y dejan atrás la actitud pasiva del que acude al aula a recibir información del docente. Se observan mayores niveles de autonomía, más momentos de toma de decisiones y una mayor responsabilidad. En este proceso, poniendo el foco en aspectos más "relacionales", el docente tiene un papel fundamental, pues para que se produzca esta independencia es necesario generar un espacio de confianza mutua que pueda amortiguar las dificultades. La postura que adopta el docente es clave para facilitar que se vayan desprendiendo de sus dudas para ir generando sus propias certezas.

Lo más importante es que el desarrollo creativo debe tener lugar en un contexto de mutuo respeto en el que los estudiantes se sientan valorados por sus ideas y contribuciones, y perciban que tienen cierto control sobre sus procesos y resultados. (PD1)

Otra de las características de esta práctica disruptiva, poniendo el foco más en "lo musical", es el uso de otro lenguaje. La mayoría de los estudiantes son consumidores de música, pero pocos han sido alguna vez intérpretes o creadores, así que el hecho de utilizar un lenguaje diferente al habitual genera confusión y resulta ser un reto añadido en la realización de la tarea.

En un principio nos sentimos algo perdidos e inexpertos, ya que ninguno de los miembros del grupo había elaborado algo parecido antes. Trabajar con la música era complicado porque nunca lo habíamos hecho, todo más libre, no había tantos límites, y eso nos hacía sentirnos inseguros. (EST21G2)

Gracias a la participación en esta actividad se promueve el aprendizaje autónomo, la responsabilidad y la búsqueda de soluciones compartida. Observamos cómo la incorporación de elementos musicales modifica sustancialmente aspectos personales, relacionales y estructurales. En el inicio de la actividad aparecen sentimientos de miedo y frustración provocados por la incertidumbre que genera esta propuesta, pero a medida que avanzan van ganando en autonomía y confianza y transforman esas emociones negativas iniciales en otras de mayor utilidad.

La frustración y demás sentimientos negativos nos suponían estar bastante tiempo parados por no saber qué hacer, cómo avanzar, hacia dónde debíamos ir. (EST10G1)

Percibimos también que la posibilidad de compartir este espacio de incertidumbre con otros facilita un sistema de cuidados que funciona de forma interdependiente e interrelacionada, donde se van generando relaciones de empatía entre los participantes. Esto permite una mayor cohesión del grupo y la alineación de los intereses individuales para llegar a una meta común compartida. Se generan rutinas de aprendizaje colaborativo reales en un contexto donde la realización de trabajos grupales es identificada como fuente de dificultades dentro de la docencia universitaria. Generalmente se abordan seccionando sus partes y realizando un reparto entre los miembros del grupo que, en última 
instancia, unen, retocan y entregan. Precisamente señalan como una de las cuestiones destacables de este proyecto la imposibilidad de trabajar en grupo como lo venían haciendo tradicionalmente, reconociendo la necesidad de poner en práctica habilidades y saberes en un espacio de trabajo cooperativo real.

Siempre he trabajado subdividiendo el trabajo y nos hemos reunido solo para unir las partes. Aquí no podíamos. Creo que aquí nadie ha dejado de aportar nunca y nadie se ha desinteresado del trabajo. (EST4G1)

El interés por el trabajo creativo dota de sentido al proceso y a la superación de las dificultades que van apareciendo, afianzando la motivación intrínseca. El miedo y la incertidumbre se van convirtiendo progresivamente en sentimientos de satisfacción. La comunicación entre los estudiantes mejora cualitativamente y el entusiasmo se contagia de unos a otros potenciando la autoestima individual y grupal.

Sin duda, el hecho de utilizar en el proceso creativo un lenguaje diferente al lenguaje verbal proporciona a los estudiantes un reto que genera incertidumbre en primera instancia, obligándoles a comunicarse con un lenguaje diferente. Y de alguna manera, esta nueva forma de intercambio ayuda a construir un espacio más democrático donde se hace necesario reinventar las dinámicas preestablecidas por las estructuras del lenguaje verbal.

Cuando empezamos la actividad estábamos muy perdidos porque ninguno de nosotros sabía música y nunca habíamos hecho nada parecido. Nos resultaba difícil crear algo sin utilizar el lenguaje verbal, estábamos tan perdidos... (EST18G2)

Además, lo musical aflora como elemento mediador para la incorporación de lo emocional y de lo anímico al proceso de aprendizaje, aspecto que se considera imprescindible para que este se produzca de manera significativa. En la interpretación grupal se requiere la presencia absoluta en ese momento, generando una atención plena y facilitando la toma de consciencia de la responsabilidad individual dentro de la colectividad.

Mientras que estábamos haciendo la creación no podíamos distraernos ni pensar en otra cosa porque si no, no salía bien. (EST37G2)

El tiempo que pasábamos haciendo esta actividad en clase me servía de desconexión, no podía distraerme con otras cosas porque si yo fallaba perjudicaba a mi grupo. (EST18G2)

Por último, en lo relativo a la evaluación, entendida esta como un proceso de investigación sobre el propio aprendizaje, se llevó a cabo de manera compartida por los y las estudiantes y las docentes. La incorporación de la investigación evaluativa en el desarrollo del proyecto proporcionó las herramientas suficientes para la reflexión compartida. Un proceso de meta-aprendizaje donde se identificaron puntos débiles y fuertes, se señalaron posibilidades de mejora y se reconocieron las bondades de los aprendizajes construidos de manera colaborativa partiendo de la creación musical.

\section{Discusión y conclusiones}

Esta investigación ilustra con evidencias de naturaleza cualitativa: a) cómo los procesos de creatividad musical colectiva pueden funcionar como elemento disruptivo en Educación Superior, al cumplir gran parte de sus características (Hedberg y Freebody, 2007); b) qué cuestiones aprenden los agentes implicados al desarrollar la práctica; y c) qué papel juega durante todo el proceso la creatividad como elemento catalizador de esta.

En lo relativo a cambios más significativos en "lo personal", podemos destacar el aumento en el compromiso activo (engagement) y la motivación que se produce en los estudiantes (Rusinek, 2005). Este tipo de prácticas favorecen la autonomía y control sobre las diferentes situaciones (Wiggins y Espeland, 2012), la alineación de los estados emocionales con el aprendizaje (Cross, Laurence y Rabionowitch, 2012), el desarrollo de la comunicación y el diálogo (Lapidaki, De Groot y Stagkos, 2012) así como la flexibilidad y la tolerancia a la frustración y a la incertidumbre (Burnard, 2012). Gracias a este aprendizaje por descubrimiento de tipo inductivo, basado en problemas estéticos de solución abierta también se activa el pensamiento divergente (Rusinek, 2005) y la capacidad de auto-diálogo crítico y reflexivo (Barnett, 1992). Se ha observado además que la experiencia ha facilitado la construcción social y significativa del conocimiento (Hedberg y Freebody, 2007).

La actividad musical propuesta se enmarca en lo que Burnard (2012) denomina creatividad colaborativa y se caracteriza por plantear procesos de responsabilidad compartida donde las ideas se generan desde situaciones de reflexión conjunta. Tal y como apunta Lapidaky, De Groot y Stagkos (2012), la creación colectiva genera una cultura del aprendizaje, de la conversación y la transgresión. Se produce el aprendizaje gracias al diálogo de múltiples voces que implica la atención de todas y cada una de ellas generando una responsabilidad mutua. Este tipo de experiencia relacional hace necesaria la interacción, el intercambio de puntos de vista, la crítica constructiva y la reconstrucción del conocimiento (Johnson y Johnson, 2004). 
Observamos cómo se han puesto en juego elementos que permiten romper con lo establecido tanto en términos "estructurales" como "relacionales" o "personales". La deslocalización espacial y temporal, la tallerización del espacio, la disolución de roles docente/estudiante, y el uso de un lenguaje artístico son también clave a la hora de caracterizar a esta práctica como disruptiva (Acaso y Manzanera, 2015).

La actividad de creación musical colectiva nos permite aproximarnos a la construcción del conocimiento desde el descubrimiento, la exploración, el diálogo y la reflexión. La música entendida como mediación genera dinámicas de trabajo grupal real donde existen responsabilidades compartidas que activan procesos de maduración social, emocional e intelectual (Lapidaky et al., 2012). Estos procesos creativos y compartidos requieren que los estudiantes negocien lo que incluyen y lo que dejan fuera, así como también tienen que decidir cómo representar de mejor manera sus ideas en modalidades y formatos múltiples. Se desarrollan capacidades de resolución de problemas al tener que tomar continuas decisiones combinando distintos modos de representación, de acuerdo con su intencionalidad y propósito colectivo (Kozma y Russell, 2005).

Otro aspecto que define esta práctica como disruptiva es la incorporación de un proceso de investigación evaluativa. La incorporación de la reflexión continua y compartida sobre la propia experiencia en la que se hacen uso los saberes disciplinares y prácticos desarrollados a lo largo de la asignatura, así como los saberes tácitos o experienciales aportados por los estudiantes supone un cambio en la manera de concebir el aula universitaria (Contreras, 2013). Es precisamente el proceso creativo el que permite el reconocimiento de esa ecología de saberes (Santos, 2010) dentro del aula, desdibujando jerarquías y democratizando el conocimiento.

El proyecto analizado vincula la experiencia musical a procesos de audición, interpretación, composición e improvisación, y pone en juego los saberes disciplinares y prácticos desarrollados a lo largo de la asignatura, así como los experienciales. Pero además de la comprensión y exploración de diferentes parámetros sonoros y elementos musicales (Lage-Gómez y Cremades-Andreu, 2018), se ha generado un cambio de la percepción acerca de las propias posibilidades creativas en relación con la composición musical, identificando todos los saberes y competencias que han desarrollado en el proceso, y produciéndose una apertura con relación a la consideración de la música dentro del sistema educativo. Si al inicio de la asignatura la mayoría la consideraban como una asignatura más del currículum, su propia experiencia creativa les ha permitido comprender las posibilidades que puede ofrecerles como docentes la música si comienzan a concebirla como una mediación educativa (Ocaña-Fernández, 2020).

Este trabajo revela el modo en que la experiencia musical permite una relación intersubjetiva que implica el intercambio cognitivo, social y emocional, y cómo ejerce una mediación en los procesos de construcción de conocimiento. Además, tal y como apunta Bresler (2005), la música supone una experiencia empática, sujeta a un tiempo y a unos ritmos que están fuera de lo apresurado del mundo circundante. La experiencia musical facilita el desarrollo de la creatividad, la flexibilidad, el pensamiento reflexivo y la comunicación (Ocaña-Fernández y Reyes-López, 2018).

Investigar este proyecto de trabajo ha permitido a las docentes articular la docencia, la investigación y la innovación, haciendo partícipes a los estudiantes de una experiencia viva que puede servirles como modelo en sus prácticas futuras, más allá de los aprendizajes propios de un currículo fragmentado con gran presencia en las etapas de la educación obligatoria y en las enseñanzas universitarias para la formación de docentes. La experiencia directa musical e investigadora ha favorecido aprendizajes más significativos, algo imprescindible para la construcción de un conocimiento capaz de generar cambios en la escuela y la sociedad.

\section{Limitaciones y futuras líneas de investigación}

Este estudio es una aproximación exploratoria inicial a prácticas docentes alternativas y disruptivas que surgen en un contexto muy determinado con unas características muy concretas. Debido al número limitado de participantes y a la naturaleza del estudio de caso, los hallazgos aquí presentados son difícilmente generalizables a otros contextos. Recomendamos realizar otros procesos de investigación tanto desde miradas cuantitativas como cualitativas, para profundizar en la potencialidad de la creación musical en el ámbito de la Educación Superior. Distintas aproximaciones sobre cómo entiende el alumnado o el profesorado estos procesos o sobre cómo se participa en estos desde otros contextos sin duda harán más profundo nuestro conocimiento sobre estas cuestiones y desembocarán en la mejora de la docencia en el ámbito universitario.

\section{Referencias bibliográficas}

Acaso, M. y Manzanera, P. (2015). Esto no es una clase. Investigando la educación disruptiva en los contextos educativos formales. Barcelona: Ariel.

Adams M., Bell, L.A. y Griffin P. (2007). Teaching for Diversity and Social Justice. New York: Routledge.

Al-Zahrani, A. M. (2015). From passive to active: The impact of the flipped classroom through social learning platforms on higher education students' creative thinking. British Journal of Educational Technology, 46(6), 1133-1148. https://doi.org/10.1111/ bjet.12353 
Anderson, T. y McCrea, R. (2012). Disruptive Pedagogies and Technologies in Universities. Educational Technology \& Society, 15(4), 380-389.

Barnett, R. (1992). Improving Higher Education. Buckingham: Open University Press.

Bauman, Z. (2007). Los retos de la educación en la modernidad líquida. Barcelona: Gedisa.

Bautista, A., Yau, X. y Wong, J. (2016). High-quality music teacher professional development: a review of the literature. Music Education Research, 19(4), 455-469. http://dx.doi.org/10.1080/14613808.2016.1249357

Botella, A.M., Hurtado, A. y Ramos, S. (2018). Innovación educativa a través de la realidad virtual y el paisaje sonoro. Creativity and Educational Innovation Review, 2, 113-127. https://doi.org/10.7203/CREATIVITY.2.13628

Bower, J. L. y Christensen, C. M. (1995). Disruptive Technologies: Catching the Wave. Harvard Business Review, 73(1), $43-53$. https://doi.org/10.1016/0024-6301(95)91075-1

Bresler, L. (2005). What musician can teach educational research. Music Education Research, 7(2), 169-183. https://doi. org/10.1080/14613800500169399

Burnard, P. (2012). Commentary: musical creativity as practice. En G. E. McPherson y G. F. Welch. (Eds.), The Oxford Handbook of Music Education, vol. 2 (pp. 21-39). New York: Oxford University Press.

Carrión, E. (2019). El uso del juego y la metodología cooperativa en la Educación Superior: una alternativa para la enseñanza creativa. Educación y Pedagogía, 23, 71-97. http://dx.doi.org/10.6035/Artseduca.2019.23.4

Contreras, J. (2013). El saber de la experiencia en la formación del profesorado. Revista Interuniversitaria de Formación del Profesorado, 27(3), 125-136.

Cross, I., Laurence, F. y Rabionowith, T.C. (2012). Empathy and creativity in group musical practices: towards a concept of empathy creativity. En G. E. McPherson y G. F. Welch. (Eds.), The Oxford Handbook of Music Education, vol. 2 (pp. 337-353). New York: Oxford University Press.

Dede, C. (2007). Transforming Education for the 21st Century. Cambridge: Harvard Education Press.

García-Gil, D. y Cremades-Andreu, R. (2019). Flipped Classroom en Educación superior. Un estudio a través de relatos de alumnos. Revista Mexicana de Investigación Educativa, 24(80), 101-123.

Hedberg, J.G. y Freebody, K. (2007). Towards a disruptive pedagogy: Classroom practices that combine interactive whiteboards with TLF digital content. Melbourne: Le@rning Federation.

Hesse-Biber, S. N. y Leavy. P. (2006). Emergent Methods in Social Research. London: SAGE.

Hutchings, M., y Quinney, A. (2015). The Flipped Classroom, Disruptive Pedagogies, Enabling Technologies and Wicked Problems: Responding to the Bomb in the Basement. Electronic Journal of E-Learning, 13(2), 106-119. Disponible en: https:// eric.ed.gov/?id=EJ1060159

Kenny, A. (2018). Exploring student learning and leadership through a university-community choral initiative. British Journal of Music Education, 35(2), 203-216. https://doi.org/10.1017/S0265051717000286

Kozma, R. y Russell, J. (2005). Modeling students becoming chemists: Developing representational competence. En J. K. Gilbert (Ed.), Visualization in science education (pp. 121-145). Dordrecht: Springer.

Johnson, D. y Johnson, R. (2004). Assessing students in groups. Promoting group responsibility and individual accountability. California: Cowin Press.

Lage-Gómez, C. y Cremades-Andreu, R. (2018). Pintando sonidos a través de la Improvisación Colectiva en Educación Secundaria, Revista Electronica Complutense de Investigación en Educación Musical - RECIEM, 15, 61-89. http://dx.doi. org/10.5209/RECIEM.55090

Lapidaky, E., De Groot, R. \& Stagkos, P. (2012). Communal creativity as sociomusical practice. En G. McPherson y G. Welch. (Eds.) The Oxford Handbook of Music Education, vol. 2 (pp. 371-388). New York: Oxford University Press.

Lorenzo-Quiles, O., Vílchez-Fernández, N. y Herrera-Torres, L. (2015). Análisis de la eficacia educativa del uso de objetos digitales de aprendizaje musical. Comparación de recursos didácticos no digitales en educación secundaria obligatoria. Infancia y Aprendizaje, 38(2), 295-326. https://doi.org/10.1080/02103702.2015.1016748

Maxwell, J.A. (2012). A realist approach for qualitative research. New York: SAGE.

Minors, H.J., Burnard, P., Wiffen, C., Shihabi, Z. y Van der Walt, J.S. (2017). Mapping trends and framing issues in higher music education: changing minds/changing practices. London Review of Education, 15(3), 457- 473. https://doi.org/10.18546/ LRE.15.3.09

Moravec, J. (2011). De la sociedad 1.0 a la sociedad 3.0. En C. Cobo y J.W. Moravec (Eds.), Aprendizaje invisible. Hacia una nueva ecología de la educación. (pp. 47-73). Barcelona: Col-lecció Transmedia XXI. Laboratori de Mitjans Interactius/Publicacions i Edicions de la Universitat de Barcelona.

Moravec, J. (2013). Knowmad society: The "new" work and education. On the Horizon, 21(2), 79-83. https://doi.org/ 10.1108/10748121311322978

Murillo-Ribes, A., Riaño-Galán, M.E. y Berbel-Gómez, N. (2018). Percepción sobre el uso de "Soundcool” como propuesta de intervención en la creación sonora y en el desarrollo de competencias docentes. Un estudio exploratorio en la formación inicial del profesorado. Psychology, Society \& Education, 10(1), 127-146. http://dx.doi.org/10.7203/LEEME.43.14007

Ocaña-Fernández, A. y Reyes-López, M.L. (2018). Ecología del aprendizaje musical: Interacciones culturales e institucionales. En Martínez Rodríguez, J.B. y Fernández Rodríguez, E. (Comps.). Ecologías del aprendizaje. Educación Expandida en contextos múltiples. Madrid: Morata.

Ocaña-Fernández, A. (2020). La experiencia musical como mediación educativa. Barcelona: Octaedro. 
Parra, F.J. y Gutiérrez, I. (2017). Implementación y análisis de una experiencia de flipped classroom en Educación Musical. Innoeduca. International Journal of Technology and Educational Innovation, 3(1), 4-14. http://dx.doi.org/10.24310/innoeduca.2017.v3i1.1964

Pérez Gómez, A. I. (2008). ¿Competencias o pensamiento práctico? La construcción de los significados de representación y de acción. En J. Gimeno (Comp.), Educar por competencias, ¿qué hay de nuevo? (pp. 59-102). Madrid: Morata.

Pérez Gómez, A. I. (2012). Educarse en la era digital. La escuela educativa. Madrid: Morata.

Perrenoud, P. (2012). Cuando la escuela pretende preparar para la vida. ¿Desarrollar competencias o enseñar otros saberes? Barcelona: Graó.

Quilty, A. (2017). Queer provocations! Exploring queerly informed disruptive pedagogies within feminist community-higher-education landscapes. Irish Educational Studies, 36(1), 125-132. https://doi.org/10.1080/03323315.2017.1289704

Rusinek, G. (2005). La composición en el aula de secundaria. Musiker. Cuadernos de música, 14, 191-208.

San Pedro, T. (2018). Abby as Ally: An Argument for Culturally Disruptive Pedagogy. American Educational Research Journal, 55(6), 1193-1232. https://doi.org/10.3102/0002831218773488

Simons, H. (2011). El estudio de caso: Teoría y práctica. Madrid: Morata.

Santos, B.d.S. (2010). Descolonizar el saber, reinventar el poder. Montevideo: Trilce.

Stake, R. (1998). Investigación con estudio de casos. Madrid: Morata

Strauss, A. y Corbin, J. (2002). Bases de la investigación cualitativa. Técnicas y procedimientos para desarrollar la teoría fundamentada. Antioquía: Universidad de Antioquía

Vass, E. (2019). Musical co-creativity and learning in the Kokas pedagogy: polyphony of movement and imagination. Thinking skills and creativity, 31, 179-197. https://doi.org/10.1016/j.tsc.2018.12.004

Wiggins, J. y Espeland, M. I. (2012). Creating in music learning contexts. En G. McPherson y G. Welch. (Eds.), The Oxford Handbook of Music Education, vol. 1 (pp.341-360). New York: Oxford University Press.

Yin, R. K. (1994). Case study research: Design and methods. Thousand Oaks: Sage. 